\title{
Agilidade em Processos de Software: Evidências Sobre Características de Agilidade e Práticas Ágeis
}

\author{
Rafael M. de Mello, Pedro C. da Silva Guilherme H. Travassos \\ Programa de Engenharia de Sistemas e Computação (PESC), COPPE/UFRJ \\ Universidade Federal do Rio de Janeiro \\ Caixa Postal 68.511 - 21.9451-970 - Rio de Janeiro - RJ - Brasil \\ \{rmaiani, ght\}@cos.ufrj.br, pedroorez@poli.ufrj.br
}

\begin{abstract}
Incorporating agility on software processes is a topic that goes beyond the discussion regarding the application of pre-defined agile methods. Aiming at strengthen evidence on the feasibility of introducing characteristics of agility and agile practices previously identified for achieving agility in software processes, this paper presents the results of a large scale survey reexecution investigating their pertinence and relevance, where the opinion of 292 subjects was gathered. The results suggest there is a consensus on the pertinence of most characteristics and practices investigated, although their relevance is not perceived in the same intensity. Additionally, new characteristics and practices were proposed.
\end{abstract}

Resumo. Incorporar agilidade aos processos de software é um tema que vai além do debate sobre a aplicação de métodos ágeis pré-definidos. Visando reforçar as evidências sobre a viabilidade de introduzir características de agilidade e práticas ágeis previamente mapeadas para obtenção de agilidade em processos de software, este artigo apresenta a reexecução em larga escala de um survey sobre a pertinência e a relevância destas características $e$ práticas, coletando a opinião de 292 participantes. Os resultados sugerem um consenso sobre a pertinência da maioria das características e das práticas investigadas, embora a relevância delas não seja percebida na mesma intensidade. Em adição, novas características e práticas foram propostas.

\section{Introdução}

A introdução de princípios de agilidade em processos de software tem sido identificada como uma das mais importantes inovações dentre as abordagens para desenvolvimento de software nos últimos anos [Vlaanderen et al., 2011]. Neste sentido, a literatura técnica apresenta iniciativas referentes à compatibilização da aplicação de métodos ágeis específicos e os níveis de maturidade do modelo MPS [Santana et al., 2006; Boria, 2013]. Entretanto, apesar de diferentes métodos ágeis serem propostos, pouco se conhece sobre como estes métodos são utilizados na prática e quais são seus efeitos no processo de software [Dybä e Dingsoyr, 2008, Stavru, 2014]. Se, por um lado, existe a percepção da inovação, por outro se revelam os riscos associados a identificar "quais são as características desejáveis em um processo de software para que este seja considerado ágil?" tendo em vista a falta de evidências sobre estas características de agilidade e sobre as práticas ágeis. 
Neste contexto, Abrantes (2012) conduziu revisões sistemáticas de literatura com o objetivo de identificar um conjunto de características de agilidade e de práticas ágeis que pudessem contribuir para a introdução de agilidade em processos de software. Como resultado, foi identificado na literatura técnica um conjunto inicial com 18 características de agilidade e 17 práticas ágeis. Visando aumentar a confiança sobre a aplicabilidade deste conjunto de características e práticas, um survey para avaliação da pertinência e da relevância destas características foi desenvolvido e executado em duas oportunidades, permitindo evoluir o conhecimento sobre as características de agilidade e práticas ágeis inicialmente estabelecidas, resultando na organização de um corpo de conhecimento sobre agilidade em processos de software [Abrantes e Travassos, 2013]. Apesar do rigor científico utilizado, as duas rodadas do survey contaram com apenas 25 participantes entre pesquisadores e profissionais de Engenharia de Software (ES), recrutados por conveniência, o que resultou em um intervalo de confiança inferior a $80 \%$, agregando-se a população e amostra de ambas rodadas. Este contexto de amostras não-probabilísticas e pequenas perante a população-alvo, frequentemente observado em surveys executados em ES, reduz a capacidade de observação, podendo levar a uma baixa confiança estatística dos resultados [de Mello e Travassos, 2013].

Desta forma, visando aumentar esta confiança, decidimos reexecutar o referido survey em larga escala, seguindo uma estratégia sistemática de recrutamento sobre grupos de uma rede social profissional, reduzindo o viés na seleção de seus membros, e aplicando-se técnicas baseadas em teoria dos grafos e análise multivariada para a estratificação destes grupos [de Mello et al., 2014, de Mello et al., 2014-2], o que nos permitiu observar a opinião de amostras representativas referentes a perfis distintos de profissionais de ES. Como resultado, esta nova execução reforçou os resultados iniciais e aumentou a confiança na pertinência da maioria das características de agilidade e das práticas ágeis investigadas, ao mesmo tempo em que nos permitiu observar com maior precisão a percepção de relevância de cada uma delas. Acreditamos que estas informações representam uma importante contribuição para a tomada de decisão pelo engenheiro de software quanto à melhoria de processos de software com foco em agilidade, não restringindo, entretanto, a necessidade de aplicar especificamente um ou outro método ágil. Adicionalmente, a amostragem em larga escala nos permitiu coletar um conjunto relevante de novas características e práticas sugeridas pelos participantes que podem representar futuras oportunidades de pesquisa.

Este trabalho está organizado em mais cinco seções. Na Seção 2 são apresentadas as principais características do protocolo do estudo. Na Seção 3 é apresentada a população do estudo e o resultado do processo da amostragem. Na Seção 4 é apresentada a análise dos resultados obtidos referentes à pertinência e à relevância das características e das práticas de agilidade investigadas, comparando-os com os resultados observados nas execuções anteriores. A Seção 5 trata das ameaças à validade do estudo. Por fim, na Seção 6 são apresentadas as conclusões.

\section{Survey em Características e Práticas de Agilidade no Processo de Software}

O survey apresentado por Abrantes (2012) tem o propósito de caracterizar a pertinência e a relevância de características e práticas para a definição de um processo de software como ágil sob o ponto de vista de profissionais de ES, no contexto dos projetos de software que adotam abordagens de desenvolvimento ágil. A Tabela 1 apresenta as 
hipóteses deste survey, estabelecidas com base no consenso entre os pesquisadores envolvidos.

Tabela 1. Hipóteses do survey [Abrantes, 2012]

\begin{tabular}{|c|c|}
\hline Hipóteses Nulas & Hipótese Alternativas \\
\hline \multirow{2}{*}{$\begin{array}{l}\text { H01- o conjunto inicial de } \\
\text { características de agilidade é } \\
\text { considerado completo, isto é, todas as } \\
\text { características de agilidade presentes } \\
\text { no conjunto inicial são pertinentes para } \\
\text { a caracterização de agilidade em } \\
\text { processos de software, e nenhuma } \\
\text { característica deve ser incluída nem } \\
\text { removida }\end{array}$} & $\begin{array}{l}\text { H1- existem características de agilidade no conjunto inicial } \\
\text { de características de agilidade que foram classificadas como } \\
\text { não pertinentes para a caracterização de agilidade em } \\
\text { processos de software. Portanto, elas precisam ser } \\
\text { removidas do conjunto inicial de características de agilidade }\end{array}$ \\
\hline & $\begin{array}{l}\text { H2- existem características de agilidade não presentes no } \\
\text { conjunto inicial de características de agilidade que foram } \\
\text { indicadas como pertinentes para a caracterização de } \\
\text { agilidade em processos de software. Portanto, elas precisam } \\
\text { ser incluídas do conjunto inicial de características de } \\
\text { agilidade }\end{array}$ \\
\hline $\begin{array}{l}\text { H02- todas as características de } \\
\text { agilidade têm o mesmo nível de } \\
\text { relevância para apoiar a inserção de } \\
\text { agilidade em processos de software }\end{array}$ & $\begin{array}{l}\text { H3- existe pelo menos uma característica de agilidade com } \\
\text { nível de relevância diferente das demais características de } \\
\text { agilidade relacionadas com a caracterização de agilidade em } \\
\text { processos de software }\end{array}$ \\
\hline \multirow{2}{*}{$\begin{array}{l}\text { H03- o conjunto inicial de práticas } \\
\text { ágeis de software está completo, isto é, } \\
\text { todas as práticas presentes no conjunto } \\
\text { inicial são relevantes ou pertinentes } \\
\text { com relação a abordagens ágeis para } \\
\text { processos de software, e nenhuma } \\
\text { prática deve ser incluída nem excluída }\end{array}$} & $\begin{array}{l}\text { H4- existem práticas no conjunto inicial de práticas ágeis de } \\
\text { software que foram classificadas como não pertinentes com } \\
\text { relação às abordagens ágeis para processos de software. } \\
\text { Portanto, elas precisam ser removidas do conjunto inicial de } \\
\text { práticas ágeis }\end{array}$ \\
\hline & $\begin{array}{l}\text { H5- existem práticas não presentes no conjunto inicial de } \\
\text { práticas ágeis de software que foram classificadas como } \\
\text { pertinentes com relação às abordagens ágeis para processos } \\
\text { de software. Portanto, elas precisam ser incluídas do } \\
\text { conjunto inicial de práticas ágeis }\end{array}$ \\
\hline $\begin{array}{l}\text { H04- todas as práticas ágeis de } \\
\text { software têm o mesmo nível de } \\
\text { relevância com relação às abordagens } \\
\text { ágeis para processos de software }\end{array}$ & $\begin{array}{l}\text { H6- existe pelo menos uma prática ágil de software com } \\
\text { nível de relevância diferente das demais práticas ágeis } \\
\text { relacionadas com abordagens ágeis para processos de } \\
\text { software }\end{array}$ \\
\hline
\end{tabular}

$\mathrm{O}$ objeto de estudo é composto de um conjunto de 16 características de agilidade e de 15 práticas ágeis apresentadas na Tabela 2, estando suas definições descritas em [Abrantes e Travassos, 2013]. Tais características e práticas foram extraídas dos resultados de revisões sistemáticas de literatura (originalmente 18 características e 17 práticas) que foram posteriormente avaliadas como pertinentes nas duas execuções anteriores deste mesmo estudo.

Tabela 2. Características de agilidade e práticas ágeis investigadas no survey.

\begin{tabular}{|c|c|c|c|}
\hline \multicolumn{2}{|c|}{ Características de Agilidade } & \multicolumn{2}{c|}{ Práticas Ágeis } \\
\hline Ser Colaborativo & Leanness & Padrões de Código & Refatoração \\
\hline Ser Cooperativo & Modularidade & Test Driven Development & Design Simples \\
\hline Ser Incremental & Ser Iterativo & Integração Contínua & Pequenas liberações \\
\hline Reflexão e Introspecção & Adaptabilidade & Metáfora & Reuniões Diárias \\
\hline Orientação a Pessoas & Equipes Pequenas & Cliente Presente & Ritmo Sustentável \\
\hline Testes Constantes & Time-Boxing & Jogo do Planejamento & Equipe Completa \\
\hline Emergência & Auto-organização & Backlog do Produto & \multirow{2}{*}{ Propriedade Coletiva } \\
de Código
\end{tabular}


Como instrumento, o estudo previu a aplicação de um questionário on-line [Abrantes e Travassos, 2013] onde os participantes devem emitir sua opinião quanto à pertinência ( $\mathrm{sim} /$ não) e quanto à relevância (não relevante/ pouco relevante/ altamente relevantel totalmente relevante) de cada característica e de cada prática apresentada na Tabela 2. Adicionalmente, o participante pôde sugerir até outras cinco características e cinco práticas, devendo também classificar, em seguida, a relevância de cada uma. A fim de avaliar as opiniões coletadas, cada participante teve sua opinião ponderada pelo seu nível de experiência, calculado com base em um conjunto de questões relacionadas à formação acadêmica e experiência prática no contexto da pesquisa. A Seção 3, a seguir, apresenta a população investigada e as amostras definidas especificamente para esta execução em larga escala.

\section{População e Amostragem}

A população do estudo foi definida a partir de uma Estratégia de Recrutamento (Rcecruitment Strategy - RS), aplicada sobre a rede social LinkedIn, apresentando significativa participação tanto de membros da academia quanto da indústria em ES. O desenvolvimento e a aplicação de RS's visa mitigar as ameaças à validade externas frequentemente relatadas em surveys e experimentos, visando ampliar a qualidade das amostras no que concerne ao sua heterogeneidade, aleatoriedade e confiabilidade. Uma descrição completa sobre a RS desenvolvida para este estudo pode ser acessada em [de Mello et al., 2014]. Outro exemplo de RS aplicada a um survey pode ser encontrado em [de Mello e Travassos, 2013].

No contexto desta RS, foi estabelecido um método de busca através do qual unidades de busca (grupos do LinkedIn) foram filtradas aplicando-se um conjunto de expressões de busca referentes ao tema de agilidade em software, sendo as unidades de busca retornadas avaliadas por um conjunto de critérios de exclusão. Em seguida, as unidades selecionadas e incluídas serviram para a composição do quadro de amostragem (sampling frame) do estudo, onde foi aplicado um método probabilístico de amostragem por agrupamento (clustering) ou estratificação. Executando-se a referida $\mathrm{RS}$, foram selecionados e incluídos 64 grupos, dentre os quais 19 grupos mais representativos disponíveis foram selecionados para compor o quadro de amostragem, contendo 202.643 membros (90\% da população total identificada nos 64 grupos). Após análise dos grupos e das características dos membros coletadas após o recrutamento [de Mello et al., 2014, de Mello et al., 2014-2], os grupos originais do LinkedIn foram reorganizados em cinco estratos distintos, apresentados nas subseções a seguir.

Nesta nova execução, optou-se por evoluir a fórmula apresentada por Abrantes e Travassos (2013) para cálculo do nível de experiência, o que resultou na seguinte fórmula (1):

$$
S(i)=(L(\text { Acad })+L(\text { AgPap })+L(\text { AgExp })+L(\text { AgProj })) / \operatorname{Max}(1),
$$

onde: Acad: nível acadêmico, escala de Likert (0-3); AgPap: número de publicações relacionadas ao contexto de agilidade em ES, escala de Likert (0-3); $A g E x p$ : experiência com métodos ágeis, escala de Likert (0-3); AgProj: número de projetos ágeis em que o participante atuou, considerando o quartil em que sua quantidade de projetos se encontra em relação à toda as unidades da amostra (0-3); Max: valor máximo do numerador (12). 
Esta evolução foi necessária para reduzir a tendência de eventual supervalorização da experiência dos participantes informando um grande número de projetos, em detrimento das demais variáveis envolvidas no cálculo.

Para fomentar uma melhor análise do perfil de cada estrato, esta execução do estudo contou ainda com a adição de um novo item no questionário original do survey: uma pergunta aberta, em que cada participante podia relatar suas "cinco principais habilidades em Engenharia de Software". Cada habilidade coletada foi codificada e organizada em grupos, conforme apresentado em [de Mello et al., 2014-2]. Conforme a ordem de resposta, cada habilidade citada recebeu uma pontuação. Neste sentido, foi observado que 20 grupos de habilidades codificadas representam mais de $90 \%$ da pontuação das habilidades relatadas pelos participantes, com destaque para habilidades pessoais $(10,56 \%)$, programação $(8,80 \%)$, análise e design de software $(8,25 \%)$, habilidades sociais $(7,78 \%)$, testes de software $(7,71 \%)$, pensamento e raciocínio $(6,24 \%)$, práticas ágeis $(5,05 \%)$, características de agilidade $(5 \%)$, requisitos de software $(4,52 \%)$ e qualidade de software $(3,65 \%)$.

\subsection{Estrato S1}

S1 é composto por sete grupos do LinkedIn que promovem debates e disseminam conceitos referentes a agilidade no processo de software, tais como gerência de projetos "ágil" e os métodos ágeis Scrum e LSD (Lean Software Development). Foi observado que o nível de experiência da amostra calculada para a distribuição de S1 é significativamente superior ao nível de experiência da amostra de S2, especialmente devido à distribuição da quantidade de projetos vivenciados pelos membros pertencentes à amostra de cada grupo. Dentre as principais habilidades relatadas pela amostra de S1, destacam-se: habilidades pessoais (11,06\%), habilidades sociais $(10,38 \%)$, análise e design de software $(9,10 \%)$ e pensamento e raciocínio $(7,52 \%)$.

\subsection{Estrato S2}

O Estrato S2 é composto por três grupos de Teste de Software, sendo que dois deles são voltados para teste "ágil", incluindo um grupo voltado para testes no contexto do método ágil XP. De modo correspondente, foi verificado que a habilidade de ES mais relevante deste estrato é teste de software (14,80\%), sendo seguido por habilidades pessoais $(9,67 \%)$, habilidades sociais $(9,67 \%)$ e características de agilidade $(8,13 \%)$. Adicionalmente, foi possível também observar uma relevante menção à qualidade de software $(5,56 \%)$, termo informalmente relacionado com testes.

\subsection{Estrato S3}

Este estrato é composto por um grupo dedicado à prática ágil de refatoração de software e por dois grupos que promovem o debate referente a testes de software, sendo um deles voltado especificamente para a prática ágil $T D D$. De fato, foi observado que as quatro principais habilidades relatadas pela amostra deste estrato têm a ver com os temas de seus grupos: programação $(15,76 \%)$, práticas ágeis $(9,43 \%)$, teste de software $(8,56 \%)$ e análise e design de software $(8,06 \%)$. 


\subsection{Estrato S4}

Este estrato é composto por três grupos exclusivamente dedicados à Gerência de Configuração de Software, o que se reflete como o principal grupo de habilidades relatadas pela amostra $(12,30 \%)$, seguida de programação $(10,73 \%)$, habilidades pessoais $(10,09 \%)$ e análise e design de software (8,20\%). Considerando-se a distribuição não-normal do nível de experiência relatado pela amostra deste grupo, foi verificado através do teste de Mann-Whitney que seu nível de experiência é significativamente inferior ao da amostra S1.

\subsection{Estrato S5}

O estrato S5 é composto por um único grupo, dedicado à Arquitetura de Software, embora o grupo de habilidades referente à arquitetura tenha recebido apenas $7,45 \%$ da pontuação total. Neste estrato, as habilidades pessoais predominam $(15,53 \%)$, seguidas por análise e design de software (14,26\%), programação $(10,21 \%)$ e pensamento e raciocinio $(9,94 \%)$.

\section{Execução e Resultados Obtidos}

Durante o período de 15 dias após o recrutamento de 7.745 membros, conforme estratégia sistemática de recrutamento apresentada em [de Mello et al., 2014, de Mello et al., 2014-2], foi possível obter respostas de 292 participantes distribuídos em seus respectivos estratos, conforme apresentado na Tabela 3, onde também é apresentado o intervalo de confiança da amostra de cada estrato calculado considerando-se o pior cenário (probabilidade de uma resposta ser atingida $=50 \%$ ) e um nível de confiança (CL) de 95\%. Deste modo, como se tratam de estratos independentes, para a interpretação dos resultados apresentados nas subseções a seguir é importante considerar a existência de diferentes intervalos de confiança para cada estrato.

Tabela 3. Amostra obtida

\begin{tabular}{|c|c|c|c|}
\hline Estrato & $\begin{array}{c}\text { Tamanho da } \\
\text { População }\end{array}$ & $\begin{array}{c}\text { Tamanho da } \\
\text { Amostra }\end{array}$ & CI para $\mathbf{C L}=\mathbf{9 5 \%}$ \\
\hline S1 & 120.315 & 97 & $9,95 \%$ \\
\hline S2 & 68.033 & 84 & $10,82 \%$ \\
\hline S3 & 9.655 & 57 & $12,94 \%$ \\
\hline S4 & 17.234 & 23 & $20,42 \%$ \\
\hline S5 & 7.335 & 31 & $17,57 \%$ \\
\hline
\end{tabular}

\subsection{Análise da Pertinência}

Para cada característica de agilidade/ prática ágil apresentada, a pertinência foi calculada através da seguinte fórmula (2) [Abrantes, 2012]:

$$
\text { Pertinence }(j)=\sum_{i=1}^{m}(\text { Answer }(i, j) * S(i))(2), \text { onde: }
$$

Pertinence $(j)$ é o valor total das respostas de todos os participantes sobre a pertinência da característica/prática j; Answer $(i, j)$ é o indicador de pertinência (1) ou não pertinência (0) definido pelo participante i para a característica/prática j; $S(i)$ é o peso atribuído ao participante $\mathrm{i} ; m$ é o total de participantes que responderam ao survey. 
Assim, para ser definida como pertinente, uma característica/prática deve atender a um valor mínimo (threshold), calculado através da seguinte fórmula (3) [Abrantes, 2012]:

$$
\text { Threshold }=0,5 * \sum_{i=1}^{m} S(i)(3) \text {, onde: }
$$

$S(i)$ é o peso atribuído ao participante $\mathrm{i} ; m$ é o total de participantes que responderam à pesquisa. Adicionalmente, conforme estabelecido no protocolo do estudo [Abrantes e Travassos. 2013], as novas características/ práticas sugeridas pelos participantes deverão ser consideradas pertinentes no caso de pelo menos dois participantes indicarem-na. Nas subseções a seguir é apresentada a avaliação da pertinência das características de agilidade e das práticas ágeis.

\subsubsection{Características de Agilidade}

Dado os resultados identificados nesta pesquisa, observou-se que, à exceção da característica Emergência no estrato S5, todas as demais características foram consideradas pertinentes em todos os estratos, o que sugere que este conjunto de características é pertinente -embora não seja possível afirmar que seja suficiente- como base para a incorporação de agilidade em processos de Software. Assim, não foram identificadas evidências suficientes para que a hipótese alternativa H1 fosse aceita. Adicionalmente, foram recuperadas 291 sugestões de características de agilidade fornecidas pelos participantes. Após a codificação e a contagem das ocorrências em cada estrato, foram identificadas cinco novas características de agilidade apresentadas na Tabela 4, com a descrição baseada nos relatos dos participantes e/ou na literatura especializada. Não foram identificadas novas características nos estratos S4 e S5, considerando-se a regra previamente estabelecida que esta deve ser sugerida por, pelo menos, dois participantes [Abrantes, 2012]. Deste modo, pôde-se refutar H01 e aceitar $\mathrm{H} 2$.

Tabela 4. Novas características de agilidades sugeridas pelos participantes.

\begin{tabular}{|c|c|c|c|c|}
\hline Característica & Descrição & S1 & $\mathbf{S 2}$ & S3 \\
\hline $\begin{array}{l}\text { Automação dos } \\
\text { Processos }\end{array}$ & $\begin{array}{l}\text { Buscar ao máximo automatizar atividades do processo de } \\
\text { software, como testes, construção e deployment. }\end{array}$ & - & (2) & (3) \\
\hline $\begin{array}{l}\text { Profissionais com } \\
\text { Habilidades } \\
\text { Múltiplas }\end{array}$ & $\begin{array}{l}\text { Equipe composta por profissionais que podem atuar diversas } \\
\text { atividades do processo, quando necessário. Assim, não há uma } \\
\text { rígida definição sobre o papel de cada membro da equipe. }\end{array}$ & - & (2) & (3) \\
\hline $\begin{array}{l}\text { Excelência da } \\
\text { Equipe }\end{array}$ & $\begin{array}{l}\text { Uma equipe composta por profissionais habilidosos e seguros } \\
\text { tende a prover agilidade ao processo de software. }\end{array}$ & (3) & - & - \\
\hline $\begin{array}{l}\text { Liderança } \\
\text { Servidora }\end{array}$ & $\begin{array}{l}\text { Filosofia antiga de liderança e resgatada nos tempos atuais que } \\
\text { envolvem o compartilhamento de poderes, colocar a } \\
\text { necessidade dos demais em primeiro lugar e ajudar as pessoas a } \\
\text { desenvolverem-se [Greenleaf, 2002]. }\end{array}$ & (3) & - & - \\
\hline $\begin{array}{l}\text { Orientação ao } \\
\text { Negócio (BVO) }\end{array}$ & $\begin{array}{l}\text { Atuar com o propósito principal de agregar valor ao negócio do } \\
\text { cliente, buscando promover um entendimento claro dos } \\
\text { stakeholders sobre como o projeto de software irá contribuir } \\
\text { para a estratégia de negócio, evitando o desenvolvimento de } \\
\text { projetos sob uma visão ambígua das necessidades de negócio. }\end{array}$ & (6) & (2) & - \\
\hline
\end{tabular}

Além das características apresentadas na Tabela 4, foi identificado ainda um conjunto de valores pessoais, que não foi incluída na lista considerando que tais valores 
estão mais relacionados ao caráter do indivíduo do que a características de um processo de software. Não obstante, alguns destes valores, tais como coragem, humildade e respeito figuram em referências sobre métodos ágeis [Larman, 2004; Beck, 2000]. Os termos inovação e planejamento também foram mencionados, mas sem que houvesse um esclarecimento direcionado para a incorporação de agilidade no processo de software.

\subsubsection{Práticas Ágeis}

A prática da metáfora foi descartada como pertinente em todas as amostras, enquanto que as demais práticas foram mantidas como pertinentes. Assim, a hipótese $\mathrm{H} 03$ foi refutada e a hipótese $\mathrm{H} 4$ aceita. Além disto, também foi observada uma tendência de rejeitar a pertinência da prática do "cliente presente", concretizada apenas nas amostras dos estratos S3 e S4. Os participantes relataram ainda 165 sugestões de práticas ágeis que, depois de codificadas, resultaram na identificação de três novas práticas pertinentes, apresentadas na Tabela 5. Deste modo, é possível aceitar também a hipótese alternativa $\mathrm{H} 5$.

Tabela 5. Novas práticas ágeis sugeridas pelos participantes.

\begin{tabular}{|c|l|c|c|c|}
\hline Característica & \multicolumn{1}{|c|}{ Descrição } & S1 & S2 & S3 \\
\hline $\begin{array}{c}\text { Behavior } \\
\text { Driven } \\
\text { Development }\end{array}$ & $\begin{array}{l}\text { Técnica na qual os testes de aceitação são definidos no início do } \\
\text { projeto do software através de linguagem natural, servindo como base } \\
\text { para a comunicação entre projetistas e stakeholders. [Soeken et al., } \\
\text { 2012] }\end{array}$ & (2) & (5) & (2) \\
\hline $\begin{array}{c}\text { Programação } \\
\text { em Pares }\end{array}$ & $\begin{array}{l}\text { Consiste na prática de dois programadores lado a lado atuando na } \\
\text { solução de um problema em uma mesma máquina, o que pode incluir } \\
\text { atividades de elaboração de algoritmos, codificação, design e teste } \\
\text { [Williams et al., 2000] }\end{array}$ & $(2)$ & $(3)$ & $(3)$ \\
\hline $\begin{array}{c}\text { Revisão de } \\
\text { Software }\end{array}$ & $\begin{array}{l}\text { Realização de atividades que incluem a revisão de código e de modelos } \\
\text { de design, o que antecipará a correção de defeitos antes da } \\
\text { apresentação/ entrega de um módulo [Wong, 2006]. Estas revisões } \\
\text { devem ser frequentes e podem ser realizadas em grupo, em pares ou } \\
\text { individualmente. }\end{array}$ & - & (4) & (3) \\
\hline
\end{tabular}

\subsection{Análise da Relevância}

Para definir o nível de relevância de uma característica de agilidade e/ou prática ágil classificada previamente como pertinente, é necessário primeiro somar as respostas de cada participante (multiplicadas por seus respectivos pesos), conforme calculado através da fórmula (4) [Abrantes, 2012] a seguir:

$$
\operatorname{Rlevel}(j)=\sum_{i=1}^{m}(\operatorname{Scale}(i, j) * S(i))(4), \text { onde: }
$$

RLevel(j) é o valor total de respostas de todos os participantes (multiplicadas por seus pesos) para a característica/prática $\mathrm{j} ; m$ é o total de participantes que responderam à pesquisa; $\operatorname{Scale}(i, j)$ é a escala de nível de relevância (0-3) definida pelo participante i para a característica/prática j; $S(i)$ é o peso atribuído ao participante i.

Nas subseções a seguir são apresentadas a análise da relevância das características de agilidade e das práticas ágeis apresentadas no survey e consideradas 
pertinentes neste estudo. Para efeito de comparação, como estão sendo analisados estratos com pesos distintos, serão apresentadas taxas de relevância ao invés de níveis de relevância, calculadas através da razão entre o nível de relevância obtido em cada característica/prática e o nível máximo de relevância possível em cada estrato.

\subsubsection{Características de Agilidade}

Através da Tabela 6, onde as características estão ordenadas pela taxa de relevância observada na maior população (S1), pode-se observar que as características $\mathrm{Ser}$ Colaborativo, Incorporação de Feedback e Adaptabilidade destacam-se por serem consideradas altamente relevantes em todas as amostras (taxa de relevância $>=75 \%$ ). Adicionalmente, embora não apresentem alta taxa de relevância em todos os estratos, ser cooperativo, ser iterativo e ser incremental também merecem destaque pela tendência a serem considerados altamente relevantes com uma baixa variância entre as amostras observadas.

Pode-se observar também que a característica orientação a pessoas é a que apresenta maior variação de taxas entre os estratos, sendo considerada altamente relevante apenas em S1, único estrato cujo perfil de principais habilidades da amostra incluem habilidades sociais. Além disto, conforme apresentado na Seção 3, este é o único estrato exclusivamente formado por apenas grupos do LinkedIn que debatem agilidade. Casos semelhantes ocorrem com as características time boxing e leanness. Deste modo, foi possível observar, através da estratificação, uma interpretação de agilidade influenciada pelo background dos participantes. Do mesmo modo, a característica emergência foi considerada de baixa relevância $(<50 \%)$ em todos os estratos onde foi avaliada, exceto em S1. A influência do background dos participantes em cada amostra também pode ser observada no caso da característica testes constantes, que foi considerada altamente relevante apenas nos dois estratos cujos membros possuem maior background em testes (S2 e S3) do que nos demais.

Tabela 6. Taxas de relevância referentes à cada característica de agilidade nos cinco estratos analisados

\begin{tabular}{|c|c|c|c|c|c|}
\hline Característica & S1 & S2 & S3 & S4 & S5 \\
\hline Ser Colaborativo & $\mathbf{8 8 \%}$ & $\mathbf{9 0} \%$ & $\mathbf{8 2} \%$ & $\mathbf{8 2} \%$ & $\mathbf{8 4 \%}$ \\
\hline Adaptabilidade & $\mathbf{8 4} \%$ & $\mathbf{7 8 \%}$ & $\mathbf{7 9 \%}$ & $\mathbf{8 0} \%$ & $\mathbf{8 1 \%}$ \\
\hline Transparência & $\mathbf{8 4 \%}$ & $\mathbf{7 8 \%}$ & $69 \%$ & $74 \%$ & $\mathbf{7 8 \%}$ \\
\hline Ser Incremental & $\mathbf{8 1 \%}$ & $73 \%$ & $\mathbf{7 8 \%}$ & $\mathbf{8 2 \%}$ & $\mathbf{8 3 \%}$ \\
\hline Incorporação de Feedback & $\mathbf{8 0 \%}$ & $\mathbf{8 1 \%}$ & $\mathbf{7 7 \%}$ & $\mathbf{8 1 \%}$ & $\mathbf{7 5 \%}$ \\
\hline Orientação a Pessoas & $\mathbf{8 0} \%$ & $70 \%$ & $59 \%$ & $54 \%$ & $52 \%$ \\
\hline Ser Iterativo & $\mathbf{7 9 \%}$ & $74 \%$ & $\mathbf{7 8 \%}$ & $\mathbf{7 5 \%}$ & $\mathbf{7 8 \%}$ \\
\hline Reflexão e Introspecção & $\mathbf{7 7 \%}$ & $70 \%$ & $70 \%$ & $56 \%$ & $66 \%$ \\
\hline Ser Cooperativo & $\mathbf{7 5 \%}$ & $\mathbf{7 6 \%}$ & $72 \%$ & $\mathbf{7 5 \%}$ & $72 \%$ \\
\hline Testes Constantes & $72 \%$ & $\mathbf{8 0 \%}$ & $\mathbf{7 7 \%}$ & $64 \%$ & $65 \%$ \\
\hline Auto-organização & $69 \%$ & $69 \%$ & $62 \%$ & $62 \%$ & $56 \%$ \\
\hline Time-Boxing & $65 \%$ & $52 \%$ & $48 \%$ & $43 \%$ & $56 \%$ \\
\hline Leanness & $61 \%$ & $43 \%$ & $47 \%$ & $53 \%$ & $45 \%$ \\
\hline Modularidade & $58 \%$ & $47 \%$ & $53 \%$ & $50 \%$ & $62 \%$ \\
\hline Equipes Pequenas & $57 \%$ & $53 \%$ & $43 \%$ & $43 \%$ & $50 \%$ \\
\hline Emergência & $54 \%$ & $36 \%$ & $45 \%$ & $44 \%$ & - \\
\hline
\end{tabular}


Portanto, apesar de haver uma grande variância de relevância para algumas características em diferentes estratos, é possível observar que existem características que tendem a ser consideradas altamente relevantes, bem como existem características que tendem a ser consideradas de baixa relevância (emergência e equipes pequenas). Deste modo, os resultados, ora apresentados, nos permitem rejeitar $\mathrm{H} 02$ e aceitar H3.

\subsubsection{Práticas Ágeis}

Através da Tabela 7, pode-se observar que poucas práticas foram consideradas altamente relevantes. De fato, apenas no caso da integração contínua, todas as amostras apresentaram uma taxa de relevância igual ou superior a $75 \%$. Entretanto, backlog do produto não é considerada uma prática altamente relevante apenas no estrato S3. Em contrapartida, as práticas do jogo do planejamento e cliente presente, tipicamente associadas aos métodos ágeis, foram consideradas práticas de baixa relevância por todos os estratos em que foram avaliadas, inclusive na amostra referente ao estrato mais diretamente relacionado às práticas ágeis. Deste modo, considerando a diversidade de relevâncias observadas, é possível rejeitar H04 aceitar H6.

Tabela 7. Taxas de relevância referentes às práticas ágeis.

\begin{tabular}{|c|c|c|c|c|c|}
\hline Prática & S1 & S2 & S3 & S4 & S5 \\
\hline Integração Contínua & $\mathbf{8 3 \%}$ & $\mathbf{8 2 \%}$ & $\mathbf{7 8 \%}$ & $\mathbf{8 1 \%}$ & $\mathbf{8 4 \%}$ \\
\hline Backlog do Produto & $\mathbf{8 3 \%}$ & $\mathbf{7 7 \%}$ & $72 \%$ & $\mathbf{7 8 \%}$ & $\mathbf{8 4 \%}$ \\
\hline Visibilidade do Projeto & $\mathbf{7 7 \%}$ & $71 \%$ & $65 \%$ & $\mathbf{7 9 \%}$ & $59 \%$ \\
\hline Pequenas liberações & $\mathbf{7 6 \%}$ & $71 \%$ & $\mathbf{7 6 \%}$ & $63 \%$ & $71 \%$ \\
\hline Equipe Completa & $74 \%$ & $73 \%$ & $57 \%$ & $71 \%$ & $55 \%$ \\
\hline Propriedade Coletiva de Código & $72 \%$ & $73 \%$ & $60 \%$ & $49 \%$ & $66 \%$ \\
\hline Ritmo Sustentável & $72 \%$ & $71 \%$ & $\mathbf{7 5 \%}$ & $\mathbf{7 6 \%}$ & $58 \%$ \\
\hline Reuniões Diárias & $71 \%$ & $68 \%$ & $65 \%$ & $70 \%$ & $59 \%$ \\
\hline Refatoração & $67 \%$ & $72 \%$ & $62 \%$ & $47 \%$ & $70 \%$ \\
\hline Design Simples & $60 \%$ & $68 \%$ & $66 \%$ & $47 \%$ & $58 \%$ \\
\hline Test Driven Development & $53 \%$ & $69 \%$ & $53 \%$ & $45 \%$ & $45 \%$ \\
\hline Padrões de Código & $53 \%$ & $62 \%$ & $52 \%$ & $43 \%$ & $46 \%$ \\
\hline Jogo do Planejamento & $42 \%$ & $48 \%$ & $37 \%$ & - & $38 \%$ \\
\hline Cliente Presente & $38 \%$ & $44 \%$ & - & - & $35 \%$ \\
\hline
\end{tabular}

Entretanto, no geral, foi observado que não há consenso entre os estratos sobre a relevância da maioria das práticas ágeis, embora seja possível perceber algumas tendências favoráveis ou contrárias a cada prática que podem estar relacionadas com as principais habilidades de cada grupo, como no caso de test driven development para S2 (69\%) e propriedade coletiva do código e pequenas liberações para S4 (49\% e 63\%, respectivamente).

\subsection{Comparação com as execuções anteriores}

Para efeitos de comparação com os resultados apresentados por Abrantes e Travassos (2013), foi realizada uma agregação simples dos resultados observados em cada estrato para cada característica, ponderada pelo tamanho da população de cada estrato, obtendo as seguintes taxas de relevância apresentadas na Tabela 8.

Deste modo, comparando-se os resultados atuais com os obtidos nas execuções anteriores, observa-se uma semelhança da relevância (alta) nas características ser 
colaborativo, adaptabilidade, incorporação de feedback, ser iterativo e ser incremental, enquanto que existe uma considerável diferença $(26,64 \%)$ nas taxas de relevância observadas para a característica transparência. A mesma agregação foi realizada para as características e a comparação pode ser visualizada na Tabela 9.

Tabela 8. Comparação entre as Taxas de Relevância- Características de Agilidade.

\begin{tabular}{|c|c|c|c|c|c|}
\hline Característica & $\begin{array}{c}\text { Estratos } \\
\text { Agregados }\end{array}$ & $\begin{array}{c}\text { [Abrantes e } \\
\text { Travassos, } \\
\mathbf{2 0 1 3}]\end{array}$ & Característica & $\begin{array}{c}\text { Estratos } \\
\text { Agregados }\end{array}$ & $\begin{array}{c}\text { [Abrantes e } \\
\text { Travassos, } \\
\mathbf{2 0 1 3}\end{array}$ \\
\hline Ser Colaborativo & $\mathbf{8 5 , 9 9 \%}$ & $\mathbf{8 4 , 7 0 \%}$ & $\begin{array}{c}\text { Testes } \\
\text { Constantes }\end{array}$ & $71,89 \%$ & $84,10 \%$ \\
\hline Transparência & $79,53 \%$ & $62,80 \%$ & $\begin{array}{c}\text { Reflexão e } \\
\text { Introspecção }\end{array}$ & $71,79 \%$ & $80,80 \%$ \\
\hline Adaptabilidade & $\mathbf{7 9 , 4 8 \%}$ & $\mathbf{8 8 , 7 0 \%}$ & $\begin{array}{c}\text { Auto- } \\
\text { Organização }\end{array}$ & $66,26 \%$ & $57,20 \%$ \\
\hline $\begin{array}{c}\text { Incorporação de } \\
\text { Feedback }\end{array}$ & $\mathbf{7 7 , 7 7 \%}$ & $\mathbf{8 4 , 1 0 \%}$ & Time-Boxing & $58,59 \%$ & $63,50 \%$ \\
\hline Ser Incremental & $\mathbf{7 6 , 1 6 \%}$ & $\mathbf{8 3 , 3 0 \%}$ & Leanness & $53,89 \%$ & $56,50 \%$ \\
\hline Ser Iterativo & $\mathbf{7 5 , 5 2 \%}$ & $\mathbf{8 4 , 8 0 \%}$ & $\begin{array}{c}\text { Equipes } \\
\text { Pequenas }\end{array}$ & $53,51 \%$ & $43,20 \%$ \\
\hline Orientação a Pessoas & $73,48 \%$ & $89,10 \%$ & Modularidade & $52,81 \%$ & $54,00 \%$ \\
\hline Ser Cooperativo & $72,67 \%$ & $79,30 \%$ & Emergência & $46,51 \%$ & $57,70 \%$ \\
\hline
\end{tabular}

No caso das práticas ágeis, é possível encontrar similaridade de resultados nas duas únicas práticas consideradas mais relevantes (integração contínua e backlog do produto) além da baixa relevância observada para Cliente Presente. Entretanto, destacam-se também as diferenças significativas de relevância observadas entre as execuções nas práticas de equipe completa $(104,33 \%)$, ritmo sustentável $(63,40 \%)$ e jogo do planejamento $(66,90 \%)$. É importante destacar ainda que, nesta nova execução, não foi possível observar a pertinência da prática da metáfora, enquanto que os participantes sugeriram a inclusão de programação em pares, prática descartada nas execuções anteriores por não ter sido considerada pertinente.

Tabela 9. Comparação entre as Taxas de Relevância- Práticas Ágeis

\begin{tabular}{|c|c|c|c|c|c|}
\hline Prática & $\begin{array}{c}\text { Estratos } \\
\text { Agregados }\end{array}$ & $\begin{array}{c}\text { [Abrantes e } \\
\text { Travassos, } \\
\mathbf{2 0 1 3}\end{array}$ & Prática & $\begin{array}{c}\text { Estratos } \\
\text { Agregados }\end{array}$ & $\begin{array}{c}\text { [Abrantes e } \\
\text { Travassos, } \\
\mathbf{2 0 1 3}]\end{array}$ \\
\hline Integração Contínua & $\mathbf{8 0 , 2 2 \%}$ & $\mathbf{9 2 , 1 0 \%}$ & $\begin{array}{c}\text { Reuniões } \\
\text { Diárias }\end{array}$ & $67,93 \%$ & $57,70 \%$ \\
\hline Backlog do Produto & $\mathbf{7 8 , 5 1 \%}$ & $\mathbf{8 2 , 7 0 \%}$ & Refatoração & $66,08 \%$ & $80,20 \%$ \\
\hline $\begin{array}{c}\text { Visibilidade do } \\
\text { Projeto }\end{array}$ & $72,46 \%$ & $75,80 \%$ & Design & $60,39 \%$ & $62,90 \%$ \\
\hline $\begin{array}{c}\text { Pequenas liberações } \\
\text { Equipe Completa }\end{array}$ & $71,99 \%$ & $66,08 \%$ & $\begin{array}{c}\text { Test Driven } \\
\text { Development }\end{array}$ & $55,72 \%$ & $45,00 \%$ \\
\hline Propriedade Coletiva \\
do Código
\end{tabular}




\section{Ameaças à Validade}

Conforme já mencionado na Seção 4, apesar da quantidade relevante de contribuições, cada estrato apresenta um tamanho de população e um tamanho de amostra específicos, o que torna a interpretação dos resultados sujeita a intervalos de confiança distintos. Como ameaça à validade interna, ressaltamos problemas de acesso ao questionário online no período em que este ficou aberto, o que pode ter impactado na participação. Adicionalmente, é importante destacar a ameaça à validade referente aos riscos operacionais envolvidos no processo de recrutamento, descritos em [de Mello et al., 2014] e no uso da fonte alternativa Linkedin como base para composição do quadro de amostragem. Finalmente, através das respostas de alguns participantes, foi possível observar uma falta de clareza na diferenciação entre os conceitos de características de agilidade e de práticas ágeis, o que os levou a sugerir práticas ágeis quando questionados sobre características e vice-versa.

\section{Conclusão}

Através do presente estudo, foi possível observar que o corpo de conhecimento proposto para a investigação de agilidade em processos de software é composto em sua grande maioria por características de agilidade e por práticas ágeis pertinentes, embora novas características e práticas tenham sido sugeridas pelos participantes. Adicionalmente, observou-se que não há um consenso sobre a relevância de diversas características e práticas investigadas. Entretanto, destacamos que características de agilidade como equipes pequenas e emergência, além de práticas ágeis como jogo do planejamento e cliente presente, tendem a ser consideradas pouco relevantes para a incorporação de agilidade no processo de software, inclusive na opinião dos participantes do estrato que apresenta o maior nível de experiência com o tema de pesquisa, desmitificando recomendações tipicamente encontradas em métodos ágeis. Em contrapartida, características como ser colaborativo, adaptabilidade e incorporação de feedback, recomendadas também externamente ao contexto dos métodos ágeis, foram consideradas altamente relevantes em todas as amostras observadas.

Deste modo, com o apoio da amostragem estratificada, foi possível observar que a resposta à questão sobre o que caracteriza agilidade em processos de software é mais complexa do que uma interpretação inicial possa sugerir, especialmente quando consideramos uma população heterogênea e representativa da comunidade de ES. Neste sentido, é importante verificar se a interpretação de agilidade feita por um indivíduo é tão somente fundamentada em uma visão estritamente pessoal de suas habilidades ou se é também fundamentada na sua observação consciente e holística do processo do estado da prática.

Como próximos passos, sugerimos a replicação deste estudo incorporando as novas características e práticas sugeridas pelos participantes. Sugerimos ainda que esta replicação inclua a mesma estratégia sistemática de recrutamento, estabelecendo novas amostras sobre a mesma população, o que poderá contribuir para a agregação de novos resultados e para aumentar a confiança nos resultados apresentados neste artigo.

\section{Agradecimentos}

Este trabalho não teria sido possível sem tomar como base os trabalhos de José Fortuna Abrantes, que em sua tese de doutorado na COPPE/UFRJ estabeleceu a estrutura 
conceitual e científica utilizada neste artigo. Prof. Travassos é bolsista de produtividade em pesquisa do CNPq.

\section{Referências Bibliográficas}

Abrantes, J. F. (2012) "Estudos Experimentais sobre Agilidade no Desenvolvimento de Software e sua Utilização no Processo de Teste" Tese de Doutorado. Programa de Engenharia de Sistemas e Computação- COPPE/UFRJ, http://www.cos.ufrj.br/uploadfile/1334062318.pdf.

Abrantes, J. F. and Travassos, G. H. (2013) "Towards Pertinent Characteristics of Agility and Agile Practices for Software Processes", In CLEI Electronic Journal 16(1).

Beck, K. (2000) "Extreme Programming Explained: Embrace Change", AddisonWesley Professional.

Boria, J. L., Rubinstein, V. L., Rubinstein, A. (2013) “A História da Tahini-Tahini: Melhoria de Processos de Software com Métodos Ágeis e Modelo MPS". Ministério da Ciência, Tecnologia e Inovação- Secretaria de Política de Informática. PBQP Software, $\quad$ http://www.softex.br/wp-content/uploads/2013/08/Livro-PBQP-SWTahini-Tahini-PT.pdf

de Mello, R. M. and Travassos, G. H. (2013) "Would Sociable Software Engineers Observe Better?" Proceedings of VII International Symposium on Empirical Software Engineering and Measurement (ESEM). IEEE.

de Mello R. M., Silva, P. C., Travassos, G. H. (2014) "Investigating Probabilistic Sampling Approaches for Large-Scale Surveys in Software Engineering". Proceedings of XVII Ibero-American Conference on Software Engineering (CIbSE), Track 11th Workshop Workshop on Experimental Software Engineering (ESELAW).

de Mello, R. M., Silva, P. C., Travassos. G. H. (2014-2) "Improving Adequacy of Software Engineering Surveys Samples by Stratifying a Professional Social Network". VIII International Symposium on Empirical Software Engineering and Measurement (ESEM). IEEE (submitted).

Dyba, T., Dingsoyr, T. (2008) "Empirical Studies of Agile Software Development: A Systematic Review". In Information and Software Technology, vol. 50, pages 833859.

Greenleaf, R., Spears, L., Covey, S. (2002) "Servant Leadership: A Journey Into the Nature of Legitimate Power and Greatness". Paulist Press.

Larman, C. (2004) "Agile and Iterative Development: A Manager's Guide", AddisonWesley Professional.

Santana, C. A., Timóteo, A. L., Vasconcelos A. M. L. (2006) "Mapeamento do modelo de Melhoria do Processo de Software Brasileiro (MPS.Br) para empresas que utilizam Extreme Programming (XP) como metodologia de desenvolvimento", Proceedings of $V S B Q S$.

Soeken, M., Wille, R., Drechsler, R. (2012) "Assisted behavior driven development using natural language processing" In Objects, Models, Components, Patterns, pages 269-287. Springer. 
Stavru, S. (2014) "A critical examination of recent industrial surveys on agile method usage". In Journal of Systems and Software, vol. 94, pages 87-97. Elsevier.

Vlaanderen, K., Jansen, S., Brinkkemper, S., Jaspers, E. (2011) "The agile requirements refinery: Applying SCRUM principles to software product management", In Information and Software Technology, vol. 53, pages. 58-70.

Williams, L. et al. (2000) "Strengthening the case for pair programming", In IEEE software, 17(4), pages 19-25.

Wong. Y. (2006) “Modern Software Review”. IGI Global. 